\title{
Research on the Bottom of the Main Sequence and Brown Dwarfs in the Early Days
}

\author{
Takenori Nakano \\ c/o Department of Physics, Faculty of Science, Kyoto University, Kyoto \\ 606-8502, Japan; E-mail: nakano@tap.scphys.kyoto-u.ac.jp
}

\begin{abstract}
One of the earliest works on the evolution of very low mass stars, Hayashi \& Nakano (1963), is introduced, which determined selfconsistently the bottom of the main sequence at $0.08 M_{\odot}$ and investigated the evolution of an object of $0.05 M_{\odot}$ from the stages with negligible electron degeneracy to those with high degeneracy.
\end{abstract}

\section{Introduction}

In addition to the well-known work by Kumar $(1963 \mathrm{a}, \mathrm{b})$, there is another work in the early days by Hayashi \& Nakano (1963, hereafter HN63) on the bottom of the main sequence and on the evolution of brown dwarfs, which seems to have almost been forgotten. HN63 extended Hayashi's (1961) work on the pre-main sequence evolution of solar mass stars to lower masses by taking into account the effects of $\mathrm{H}_{2}$ molecules, e.g., the partial dissociation of $\mathrm{H}_{2}$, to the structure of the stellar atmospheres.

\section{The Bottom of the Main Sequence}

To determine the zero-age main sequence (ZAMS) stages, we have to know the energy release rate $L_{\mathrm{H}}$ by hydrogen burning in the stars and the energy loss rate $L$ from the stellar surfaces, that is determined by the radiative/convective energy transport in the stars. Adopting a polytropic structure of index 1.5 for stars of mass $M \lessgtr 0.26 M_{\odot}$, HN63 calculated $L_{\mathrm{H}}$ as a function of the stellar radius $R$ for a given $M$. Because these stars can be regarded as fully convective except for the very thin surface layers, $L$ is determined by the structure of the atmospheres. By investigating the atmospheric structure, HN63 determined the effective temperature $T_{\mathrm{e}}$ of the star as a function of $R$, and obtained $L$. By comparing $L_{\mathrm{H}}$ with $L$, they determined the stage at which the quasi-steady state $L_{\mathrm{H}}=L$ is first realized (ZAMS) as shown in Fig. 1. They found that the quasi-steady state is not realized for $M<0.08 M_{\odot}$ because of too low central temperatures. They also investigated the depletion of lithium during the contraction, which can offer a test for being brown dwarfs (e.g., Rebolo, Martín, \& Magazzù 1992).

Although Kumar (1963a) determined $L_{\mathrm{H}}$ in a similar way, he adopted for $L$ the reasonable values suggested by the luminosities of Ross 614B and L726-8, assuming that these stars were close to the bottom of the main sequence. 


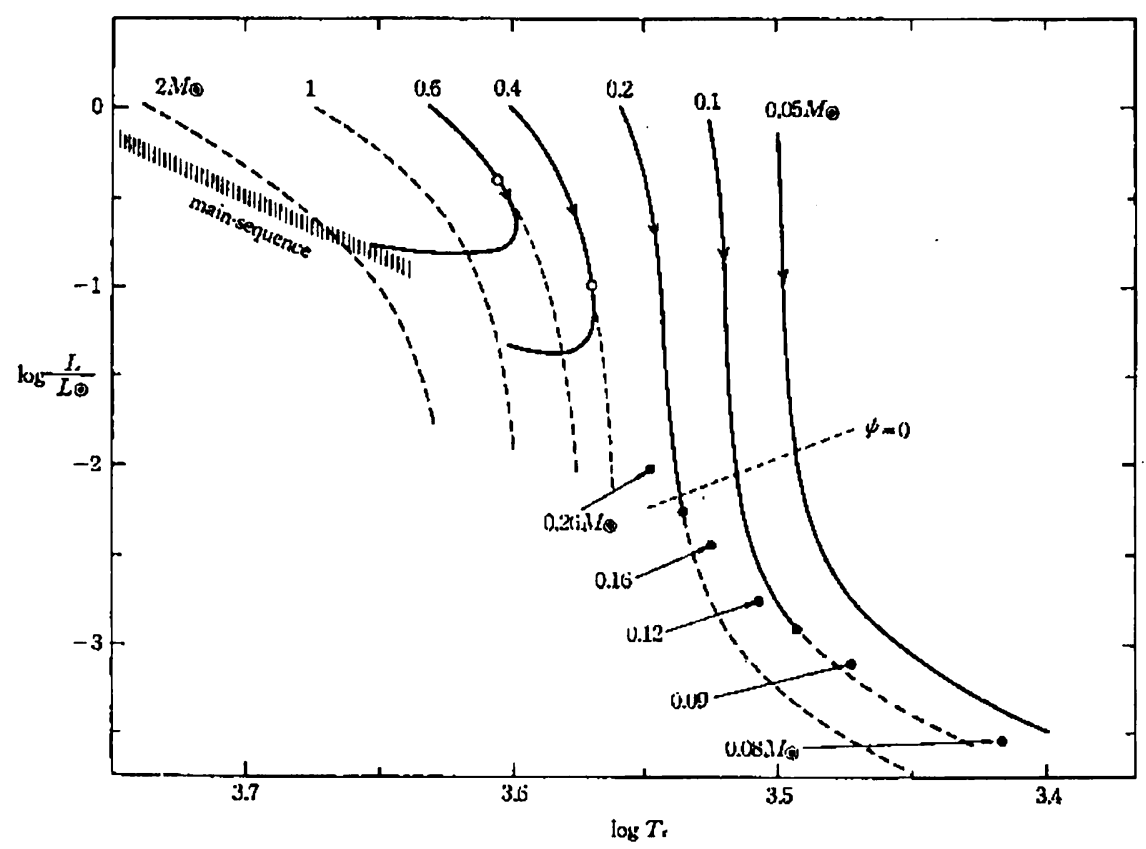

Figure 1. The evolutionary paths (solid lines) and the zero-age main sequence stages (filled circles) on the HR diagram (Fig. 2 of HN63).

\section{Evolution of Brown Dwarfs}

Kumar (1963b) estimated the contraction time of substellar objects assuming $T_{\mathrm{e}}$ to be constant during the contraction. This assumption approximately holds when the electron degeneracy has little effect (e.g., Fig. 1). When the degeneracy has a great effect, $R$ is kept nearly constant instead of $T_{\mathrm{e}}$. HN63 investigated the evolution of a $0.05 M_{\odot}$ object from the stages with negligible electron degeneracy to those with high degeneracy. Determining $T_{\mathrm{e}}$ in the way described above, HN63 obtained the evolutionary path on the HR diagram as shown in Fig. 1.

Acknowledgments. I am grateful to E. L. Martín, M. Tamura, A. Tokunaga, and T. Tsuji for their kind consideration to realize this article.

\section{References}

Hayashi, C. 1961, PASJ, 13, 450

Hayashi, C., \& Nakano, T. 1963, Progr. Theor. Phys., 30, 460 (HN63); or http://www-tap.scphys.kyoto-u.ac.jp/ nakano/member-e.html

Kumar, S. S. 1963a, ApJ, 137, 1121

Kumar, S. S. 1963b, ApJ, 137, 1126

Rebolo, R., Martín, E. L., \& Magazzù, A. 1992, ApJ, 389, L83 